(6) OPEN ACCESS

- Additional material is published online only. To view please visit the journal online (http://dx.doi.org/10.1136/ bjophthalmol-2017-311328).

${ }^{1} \mathrm{UCL}$ Institute of Ophthalmology, University College London, London, UK ${ }^{2}$ Moorfields Eye Hospital NHS Foundation Trust, London, UK ${ }^{3}$ Department of Ophthalmology and Visual Sciences, Medical College of Wisconsin,

Milwaukee, Wisconsin, USA ${ }^{4}$ Department of Ophthalmology, Stanford University, Palo Alto, California, USA

\section{Correspondence to} Michel Michaelides, UCL Institute of Ophthalmology, University College London, London EC1V 9EL, UK; michel. michaelides@ucl.ac.uk

Received 15 September 2017 Revised 23 October 2017 Accepted 4 November 2017 Published Online First 15 November 2017

Check for updates

To cite: Georgiou M, Kalitzeos A, Patterson EJ, et al. Br J Ophthalmol 2018;102:1028-1035.

\title{
Adaptive optics imaging of inherited retinal diseases
}

\author{
Michalis Georgiou, ${ }^{1,2}$ Angelos Kalitzeos, ${ }^{1,2}$ Emily J Patterson, ${ }^{3}$ Alfredo Dubra, ${ }^{4}$ \\ Joseph Carroll, ${ }^{3}$ Michel Michaelides ${ }^{1,2}$
}

\begin{abstract}
Adaptive optics (AO) ophthalmoscopy allows for noninvasive retinal phenotyping on a microscopic scale, thereby helping to improve our understanding of retinal diseases. An increasing number of natural history studies and ongoing/planned interventional clinical trials exploit AO ophthalmoscopy both for participant selection, stratification and monitoring treatment safety and efficacy. In this review, we briefly discuss the evolution of AO ophthalmoscopy, recent developments and its application to a broad range of inherited retinal diseases, including Stargardt disease, retinitis pigmentosa and achromatopsia. Finally, we describe the impact of this in vivo microscopic imaging on our understanding of disease pathogenesis, clinical trial design and outcome metrics, while recognising the limitation of the small cohorts reported to date.
\end{abstract}

\section{INTRODUCTION}

Inherited retinal disease (IRD) is the leading cause of legal blindness in England and Wales among the working age population and the second most common in childhood. ${ }^{1}$ IRD is a group of clinically heterogeneous conditions, which can result in diagnostic challenges, often thereby necessitating detailed multimodal retinal imaging, as well as electrophysiological and psychophysical evaluation. They are subject to a broad range of research avenues and interventions which have been recently reviewed. ${ }^{2}$ Here, we categorise IRDs on the basis of natural history (stationary or progressive) and the primarily affected retinal cell type.

In vivo retinal imaging has been rapidly evolving over the last decades primarily due to advances in optics, electronics and computer technology. The introduction of optical coherence tomography (OCT) has revolutionised the clinical investigation of retinal diseases. ${ }^{34}$ One of the main limiting factors for in vivo retinal imaging is ocular aberrations, due to the optical imperfections of the eye. ${ }^{5}$ Adaptive optics $(\mathrm{AO})$ can be employed in ophthalmology to overcome the aforementioned limitation. ${ }^{6}$

\section{BRIEF OVERVIEW OF AO RETINAL IMAGING}

The incorporation of AO to any ophthalmoscopic technique, including fundus photography, OCT and scanning laser ophthalmoscopy (SLO), provides in vivo microscopic imaging. ${ }^{6-9} \mathrm{AO}$ ophthalmoscopes typically use a wavefront sensor to measure the ocular monochromatic aberrations and a deformable mirror to correct for the detected aberrations. ${ }^{6910}$ Herein we will be focusing on AOSLO photoreceptor imaging as this is the modality that has been most extensively used in patients with IRD. By focusing a scanning light source on the photoreceptor layer and rejecting out-of-focus light through the use of a confocal aperture, axial sectioning is achieved, thereby increasing image contrast. ${ }^{8} 11$ Photoreceptors with relatively intact outer segments waveguide some incident light, and backscatter a very small fraction (less than $0.1 \%$ ), which is used for imaging. ${ }^{12}$ When collecting that light in a confocal detector, the cone ${ }^{81314}$ and perifoveal $\operatorname{rod}^{81516}$ mosaics can be resolved. Several systems have been developed including both custom-built and commercially available devices.

The non-confocal backscattered light can also be exploited to reveal the photoreceptor inner segment mosaic. For example, the split detection (SD) technique (SD-AOSLO) does so by subtracting images created by capturing the light to the left of the confocal aperture with one detector and the light to the right of it with a different one. ${ }^{17}$ This recent development was transformational because cones with compromised outer segments (as would be anticipated in the majority of IRDs) can now be reliably identified for the first time. This has major implications for patient stratification and targeting of intervention. ${ }^{18-25}$

Due to light safety restrictions, each individual AOSLO raw frame is captured using very low illumination power $(\sim 100 \mu \mathrm{W}$ at the pupil) and thus the resulting images are inherently noisy. Therefore, AOSLO image sequences are captured at each retinal location of interest, and used to create a higher signal-to-noise ratio (SNR) image by averaging a few of these frames after correcting for eye motion and scanning distortions. ${ }^{26}$ These high SNR images are then stitched together to create a larger montage (figure 1). A range of photoreceptor metrics have been employed to date, with cone density for a given eccentricity being the most widely used, and usually compared with normative data from histology ${ }^{27}$ or imaging studies. ${ }^{13} 2829$ Other metrics include (1) cone spacing-average distance between cells in a given location, (2) Voronoi analysis (figure 1) which involves counting the number of neighbouring cells based on the distance between them, thereby assessing mosaic geometry, ${ }^{28}$ (3) reflectivity, ${ }^{18}$ and (4) metrics for the preferred orientation of cones and local spatial anisotropy. ${ }^{30}$

The combination of OCT and AO (AO-OCT) is an evolving field, aiming for $3 \mathrm{D}$ reconstruction and offers greater axial resolution compared with AOSLO. ${ }^{31}$ 


\section{IRDS AND AO RETINAL OPHTHALMOSCOPY}

The selected conditions below have been prioritised based on the ability of published AO ophthalmoscopy studies to demonstrate clinical, research or trial utility. There are inherent limitations due to the often small cohorts reported to date. These are usually small due to the vast genetic and phenotypic heterogeneity of IRDs, the low prevalence of each genotype and due to the difficulty of establishing multicentre studies given the limited availability of AOSLO. However, similar limitations are often faced by other studies using other modes of high-resolution imaging. For clarity, we have included the number of subjects in each study we describe and whether the patients were molecularly confirmed (online supplementary table 1 ).

\section{Macular dystrophies}

Stargardt disease

Stargardt disease (STGD1) is the most common form of hereditary macular dystrophy. ${ }^{32}$ Confocal AOSLO (cAOSLO) has demonstrated abnormal and decreased cone spacing in regions corresponding to areas of reduced and irregular fundus autofluorescence (FAF), in predominantly late-onset/foveal sparing molecularly proven patients (11 of 12 patients). ${ }^{33}$ Moreover, foveal retinal pigment epithelium (RPE) cells were imaged in areas where the photoreceptor mosaic appeared disrupted in confocal reflectance imaging, suggesting photoreceptor loss preceding RPE cell loss-although the application of SD-AOSLO would address whether there are in fact cone inner segments present. ${ }^{33}$ Song et al also reported increased photoreceptor spacing, in genetically proven STGD1 $(n=2)$, in otherwise normal appearing areas on OCT and FAF imaging; also consistent with photoreceptor loss preceding clinically detectable RPE disease. ${ }^{34}$ Interestingly, SD-AOSLO derived cone density has been shown to correlate well with OCT measurements of outer nuclear layer thickness and retinal sensitivity $(n=14$; all molecularly confirmed), demonstrating a valuable structure-function association, even though the extent of atrophic changes was not corresponding to visual aquity. ${ }^{19}$ Using cAOSLO and SD-AOSLO, Tanna et al investigated the reliability and repeatability of cone counting in patients with STGD1 $(n=12)$, suggesting superior reliability and repeatability with SD-AOSLO. ${ }^{35}$

Longitudinal imaging studies of the photoreceptor and RPE mosaic in large molecularly proven specific STGD1 cohorts (ie, childhood-onset, adult-onset and late-onset/foveal sparing) are needed to evaluate cellular disease progression and potentially identify the most suitable participants for ongoing and multiple planned gene therapy and pharmacological interventions. ${ }^{1933} 3436$ AO ophthalmoscopy may be a useful method of monitoring in trials, since 'classic' parameters of ophthalmological examination including best corrected visual acuity (BCVA) are not sufficiently sensitive outcome measures for conditions such as STGD1. ${ }^{37}$

\section{Best disease}

Normal photoreceptor structure and cone densities in areas adjacent to clinically visible lesions have been reported, with persistent photoreceptor structure overlying stage 1 and 2 vitelliform Best disease lesions, in keeping with relatively intact visual acuity (VA). ${ }^{38}$ Using cAOSLO and SD-AOSLO, variable photoreceptor architecture has been observed to be associated with different stages of the disease and the location within the lesions, including reduced cone density, due to major discontinuities/gaps in the mosaic, and cone inner segment enlargement. ${ }^{23}$

\section{X-linked retinoschisis}

Duncan et $a l^{39}$ have reported increased and irregular cone spacing within the foveal schisis characterising X-linked retinoschisis. Interestingly, cone spacing was normal and regular elsewhere. The preserved waveguiding cones at the fovea and eccentric macular regions may indicate increased likelihood of successful rescue with intervention-and could also be helpful in patient selection.

\section{Stationary dysfunction syndromes}

Cone dysfunction syndromes

This group of disorders has been reviewed in detail previously ${ }^{40}$ (figure 2).

\section{Achromatopsia}

Early investigations with cAOSLO identified 'dark spaces' in the cone mosaic, increased cone spacing and/or decreased cone density in patients with achromatopsia (ACHM) 16184142 (figure 2: 1C and 2C). Marked variability in the cone mosaic has been observed across patients; with no significant difference between the two most common genotypes, CNGA3 and $\mathrm{CNGB}^{42}{ }^{43}$; and the rarer GNAT2 genotype associated with
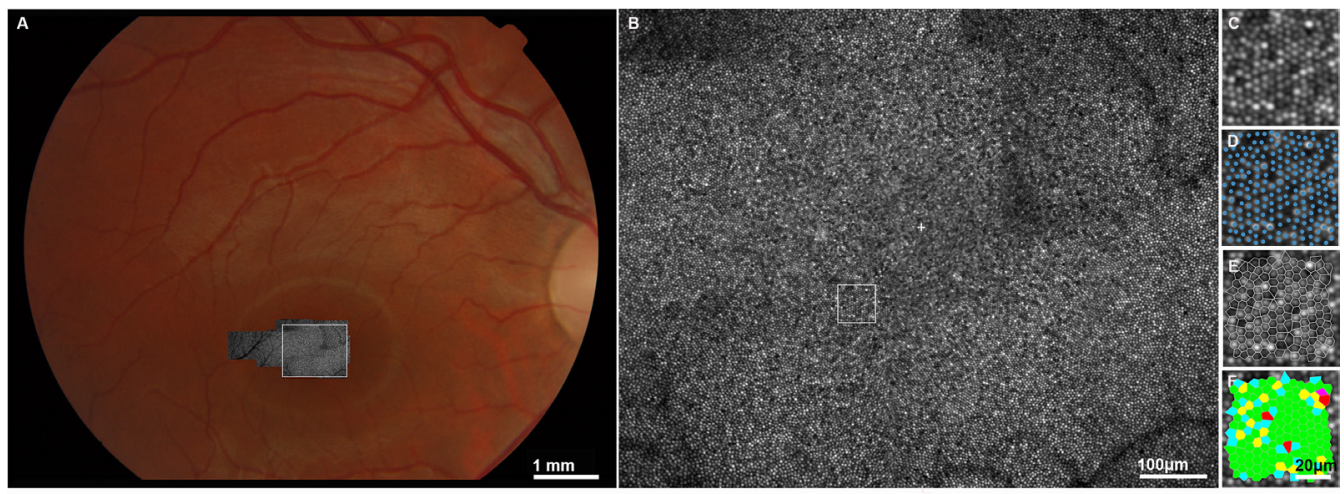

Figure 1 Adaptive optics scanning laser ophthalmoscopy (AOSLO) imaging of a healthy subject and cone quantification. (A) Colour fundus photograph $\left(30^{\circ}\right)$ of a healthy subject (MM_0136), with AOSLO montage superimposed. The white square encompasses the foveal avascular zone (region of interest, ROI), which is magnified in (B). (B) Confocal AOSLO of the ROI, the estimated foveal centre is marked with a white cross and the $55 \mu \mathrm{m} \times 55 \mu \mathrm{m}$ area of sampling for cone counting with a white box at $0.35^{\circ}$ from the foveal centre. Scale bar $=100 \mu \mathrm{m}$. (C) Magnified view of the sampled area. (D) The sampled area with cones marked. (E) The sampled area with Voronoi domains. (F) The Voronoi representation coloured according to the number of neighbouring cells. Green represents six-sided bound cones. Scale bar for $(C-F)=20 \mu \mathrm{m}$. 

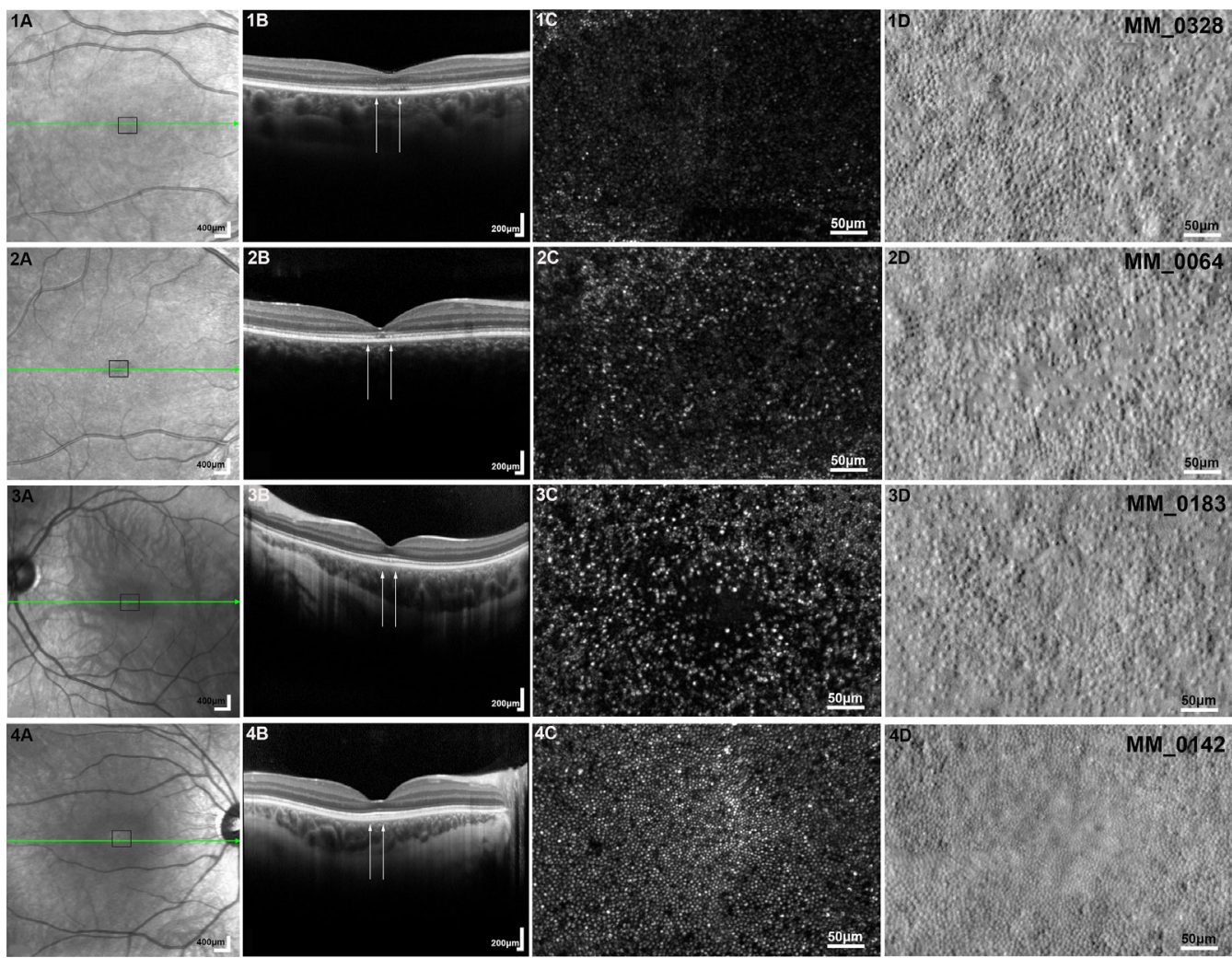

Figure 2 Adaptive optics scanning laser ophthalmoscopy (AOSLO) imaging of the cone dysfunction syndromes. Column (A) shows the infrared reflectance (IR) fundus photographs for each subject $(1,2,3,4)$. The green arrow represents the section in which the optical coherence tomography (OCT) (Spectralis HRA+OCT, Heidelberg Engineering, Heidelberg, Germany) presented in column (B) is taken; the black square represents the 450 $\mu \mathrm{m} \times 300 \mu \mathrm{m}$ region of interest imaged with AOSLO which is presented in columns (C) and (D). Column (B) shows OCT horizontal scans through the fovea and the white arrows mark the corresponding AOSLO area (450 $\mu \mathrm{m}$ wide). Column (C) depicts confocal AOSLO (cAOSLO) and column (D) split detection (SD) AOSLO. Subjects (1) and (2) have achromatopsia associated with CNGB3 and CNGA3 gene mutations, respectively. (1C/2C) Dark spaces are observed, due to loss of cone waveguiding properties, which correspond to visible foveal cone inner segments in (1D/2D), respectively, with a substantial difference in cone numerosity between the two subjects. (3) A molecularly confirmed subject with blue cone monochromacy. (3C) Dark foveal centre, with a sparse array of large bright spots, which are believed to be $S$ cones, immediately surrounding it. (3D) Remnant inner segment structure. (4) A molecularly confirmed subject with Bornholm eye disease (LIAVA haplotype). (4C) All cones are resolved in cAOSLO, with a few apparent non-waveguiding cones (dark spaces). (4D) SD-AOSLO does not resolve foveal inner segments due to the better preserved mosaic (smaller cone diameters and tighter packing geometry) compared with the other cone dysfunction syndromes. All AOSLO images were acquired using a custom-built AOSLO housed at University College London/Moorfields Eye Hospital, London. Scale bar=50 $\mu \mathrm{m}$.

the relatively least disrupted photoreceptor mosaic. ${ }^{18} 44$ Until the advent of SD-AOSLO, it was unknown if these 'dark spaces' harboured non-waveguiding cones or indicated loss of coneswith the presence or absence of cones being directly related to potential rescue with intervention. Simultaneous cAOSLO and SD-AOSLO have allowed the identification of cone inner segment structure in these spaces, ${ }^{17242545}$ with transformational implications on our understanding of ACHM and participant selection for ongoing CNGA3-ACHM and CNGB3-ACHM gene therapy trials (figure 2: $1 \mathrm{D}$ and $2 \mathrm{D}$ ). Given the potential disconnect between OCT and AO measures of cone integrity and the ability of AO to directly visualise the target cones for gene replacement, non-confocal SD-AOSLO imaging would be the modality of choice to identify patients most likely to benefit from cone-directed rescue.

In the largest AO ophthalmoscopy ACHM study to date $(n=52)$, significantly decreased peak foveal cone densities and increased spacing, using SD-AOSLO in CNGB3-ACHM has been reported. ${ }^{45}$ Interestingly, the peak foveal density ranges were shown to overlap between the previously described OCT grades, ${ }^{43}$ in keeping with the aforementioned disconnect.
Reduced reflectivity in the majority of residual cones in CNGA3 and CNGB3 has also been noted, with relative preservation in GNAT2. Changes in cone reflectivity could potentially provide a clinical trial outcome metric. ${ }^{1842}$

Directly relevant to the ongoing debate on whether ACHM is significantly progressive, ${ }^{40}$ based on serial OCT and AOSLO, a longitudinal study of CNGB3-ACHM, with follow-up of 6-26 months, showed little or no detectable change in foveal cone structure over time. ${ }^{24}$

\section{Blue cone monochromacy}

Blue cone monochromacy (BCM) is associated with a range of opsin array genotypes, affecting both $\mathrm{L}$ and $\mathrm{M}$ cones. ${ }^{40}$ The condition is X-linked, and despite female carriers being asymptomatic, cAOSLO has demonstrated variably reduced cone density, increased spacing and disrupted organisation, with phenotypic variability likely relating to random X-chromosome inactivation. ${ }^{46}$ Affected men have a more severe phenotype, although the degree of cone mosaic disruption is also highly variable and may be partly related to specific genotype group. ${ }^{47}$ The 
L/M interchange haplotypes have been associated with significantly greater residual parafoveal cone structure, with localised loss of waveguiding cones at the fovea. In contrast, the inactivating Cys203Arg missense mutation genotype group is associated with greater loss of waveguiding $\mathrm{L} / \mathrm{M}$ cones. cAOSLO images of the cone mosaic typically show a dark foveal centre, with a sparse array of large bright spots, which are believed to be $\mathrm{S}$ cones, immediately surrounding it (figure $2 \mathrm{C}$ ) ${ }^{47}{ }^{48} \mathrm{It}$ is possible to estimate with reasonable confidence the number of $\mathrm{L}$ and $\mathrm{M}$ cones in the parafovea, as they appear as dark gaps within the rod mosaic. ${ }^{46}$ cAOSLO has demonstrated a reduced number of cones in the parafovea (both reflective $S$ cones and non-reflective $\mathrm{L}$ cones and $\mathrm{M}$ cones) to that of about $25 \%$ of normal, with evidence of even greater loss of cone cells in the locus control region deletion genotype group of BCM. Moreover, SD-AOSLO images have revealed remnant inner segment structure (figure $2 \mathrm{~B}$ ) both at the fovea and the parafovea. ${ }^{48}$ Importantly, however, despite low cone density in BCM, the number is higher than that expected for the $S$ cone submosaic, in keeping with remnant $\mathrm{L} / \mathrm{M}$ cones. ${ }^{27} 4950$

Overall, these AOSLO imaging studies have identified significant intersubject variability in cone mosaic integrity and illustrate the importance of cellular imaging in the identification of remnant cones that have the potential to be rescued in planned interventions.

\section{Oligocone trichromacy and RGS9/R9AP-associated retinopathy ('Bradyopsia')}

The cone photoreceptor mosaic in three patients with typical oligocone trichromacy has been investigated, and in keeping with the original disease mechanism hypothesis, a decreased number ('oligocone') of otherwise normal appearing foveal cones (thereby permitting 'trichromacy') were observed, with absence of visible structure beyond the central fovea. ${ }^{51} R G S 9 / R 9 A P$-associated retinopathy is clinically indistinguishable from oligocone trichromacy, but can be discerned using non-standard extended electrophysiological assessment or molecular genetic testing. However, unlike in oligocone trichromacy, cAOSLO has revealed a normal cone photoreceptor mosaic in subjects with RGS9/R9AP retinopathy, ${ }^{51}{ }^{52}$ which is in agreement with the electroretinography (ERG) findings of normal initial response in dark-adapted flicker ERGs performed with a dim stimulus. ${ }^{53}$ Cellular phenotyping is therefore able to readily differentiate between these two conditions with common clinical featureswith an intact photoreceptor mosaic in bradyopsia and disruption in oligocone trichromacy.

\section{Bornholm eye disease}

Similar to BCM, Bornholm eye disease (BED) is an X-linked cone dysfunction syndrome that is associated with mutations in the L/M gene array. ${ }^{54-58}$ Predominantly due to the heterogeneity in the underlying genotype (predominantly $\mathrm{L} / \mathrm{M}$ interchange haplotypes), the degree of photoreceptor mosaic disruption in affected men is highly variable, with cone density ranging from near normal to more than $75 \%$ reduction ${ }^{59}$ (figure 2: $4 \mathrm{C}$ and 4D). However, there is also high variability in the appearance of the cone mosaic within brothers who share the same genotype, likely owing to variations in L:M cone ratio. ${ }^{5960}$ Cone density has been found to correlate with both axial length and the degree of myopia ${ }^{60}$; however, systematic analysis of the relationship between these factors and the specific underlying $\mathrm{L} / \mathrm{M}$ opsin variant is lacking, due to small numbers of subjects within each genotype group to date. Additionally, previous investigations employing AOSLO imaging have been cross-sectional, so there is a need for longitudinal studies to track larger genetically confirmed cohorts, both for BED and BCM, to determine natural history and thereby better establish the potential for intervention.

\section{Rod dysfunction syndromes}

\section{Congenital stationary night blindness}

Godara et al reported retinal structure in three patients with GRM6-associated congenital stationary night blindness. ${ }^{61}$ They identified a contiguous cone mosaic and normal cone densities with cAOSLO, in keeping with previous histopathology. They identified photoreceptor mosaic integrity and reported thinning of inner retinal layers on OCT, suggesting a functional defect in retinal neurotransmission, rather than a structural photoreceptor defect. ${ }^{61}$

\section{Oguchi disease}

Oguchi disease is a very rare form of night blindness having the unusual distinguishing features of the Mizuo-Nakamura phenomenon: diffuse fundus discoloration and return to normal colour after prolonged dark adaption. ${ }^{62}$ To probe the underlying basis of this intriguing phenomenon, the photoreceptor mosaic has been investigated, both in light and dark-adapted conditions, in two molecularly confirmed siblings. ${ }^{61}$ Normal photoreceptor densities were identified; however, rod reflectivity (unlike cone) was shown to increase over time, changing from scotopic to photopic conditions, suggesting that rods are responsible for the unique fundus findings in Oguchi disease. ${ }^{63}$

\section{Fundus albipunctatus}

Using fluorescence AOSLO and cAOSLO, Song et al have reported decreased foveal cone density and increased cone spacing at $10^{\circ}$ of eccentricity, despite this predominantly being a rod disorder. ${ }^{64}$ No photoreceptors or RPE cells were visualised within the albipunctate spots. Another study also identified decreased perifoveal cone density and mosaic disruption using cAOSLO in $R D H 5$-associated fundus albipunctatus. ${ }^{6 .}$

\section{Progressive retinal dystrophies}

Rod-cone dystrophies

Non-syndromic retinitis pigmentosa

In X-linked RPGR-associated retinopathy, there is a phenotypic heterogeneity (both intrafamilial and interfamilial) among affected men. ${ }^{66}$ Female carriers' phenotypes can vary widely, ranging from asymptomatic to severely affected (although not to the extent of affected men) and almost always present with a radial pattern of increased retinal reflectivity, the so-called tapetal-like reflex. ${ }^{67}$ The patchy appearance of rods and cones observed both ex vivo and in vivo (mosaicism) is believed to be due to random X-chromosome inactivation.

Several studies have reported a decrease in cone density and/ or increased cone spacing using cAOSLO in patients with retinitis pigmentosa (RP), with approximately half of the subjects having an established genetic diagnosis ${ }^{68-73}$ (figure 3). Sun et al ${ }^{21}$ examined both patients with RP and Usher syndrome (USH) (see below) using cAOSLO and SD-AOSLO, and found that foveal cone density was reduced by up to $38 \%$ before VA was affected, without any visible findings on OCT (however, increased cone spacing was not identified in isolated RP cases). This was in keeping with a previous study reporting normal VA and retinal sensitivity in patients with up to $62 \%$ reduction in peak cone density. ${ }^{73}$ These studies illustrate the remarkable redundancy in 
cone populations, the importance of multimodal imaging and the disconnect between retinal structure and function, with major implications for gene therapy, and also stem cell replacement strategies-including the potential need to successfully integrate smaller numbers of cones than previously believed.

A phase II/III trial has been undertaken with intravitreal implants of encapsulated human RPE cells engineered to continuously secrete ciliary neurotrophic factor (CNTF) protein in patients with early-stage and late-stage RP. ${ }^{74}$ Patients were randomly assigned to receive a high-dose or low-dose implant in one eye and sham surgery in the fellow eye. Primary endpoints were change in BCVA at 12 months for late-stage RP and change in visual field sensitivity at 12 months for early RP. Neither study showed a therapeutic benefit. However, a pilot study using AOSLO in three patients with CNTF implants over a 24-month period found that cone density remained stable in eyes with a CNTF implant, whereas there was continued cone loss in untreated fellow eyes, suggesting that more sensitive metrics are needed as primary outcome measures in progressive diseases such as $\mathrm{RP}^{71}$

\section{Usher syndrome}

AOSLO has been previously undertaken in one patient with USH type II, and three patients with USH type III. ${ }^{71}{ }^{75}$ In USH-III, a relative preservation of foveal cone density was observed, with loss of cone structure in areas of absent retinal sensitivity. ${ }^{75}$ Using CAOSLO and SD-AOSLO as complementary modalities, Sun et $a l^{21}$ identified lower foveal and parafoveal cone densities in USH-II ( $\mathrm{n}=4, \mathrm{USH} 2 \mathrm{~A}$ ) compared with non-syndromic RP ( $\mathrm{n}=9$ (2 X-linked RP RPGR; 3 autosomal recessive $\mathrm{RP}=2$ USH $2 A$ and 1 EYS; 4 autosomal dominant $\mathrm{RP}=3$ RHO and $1 \mathrm{RP} 1)$ ) despite the normal appearance of interdigitation (IZ) and ellipsoid zones (EZ) on OCT, which was attributed by the authors to the decreased number of normal waveguiding cones (outer segment defects), possibly a result of the different molecular pathways

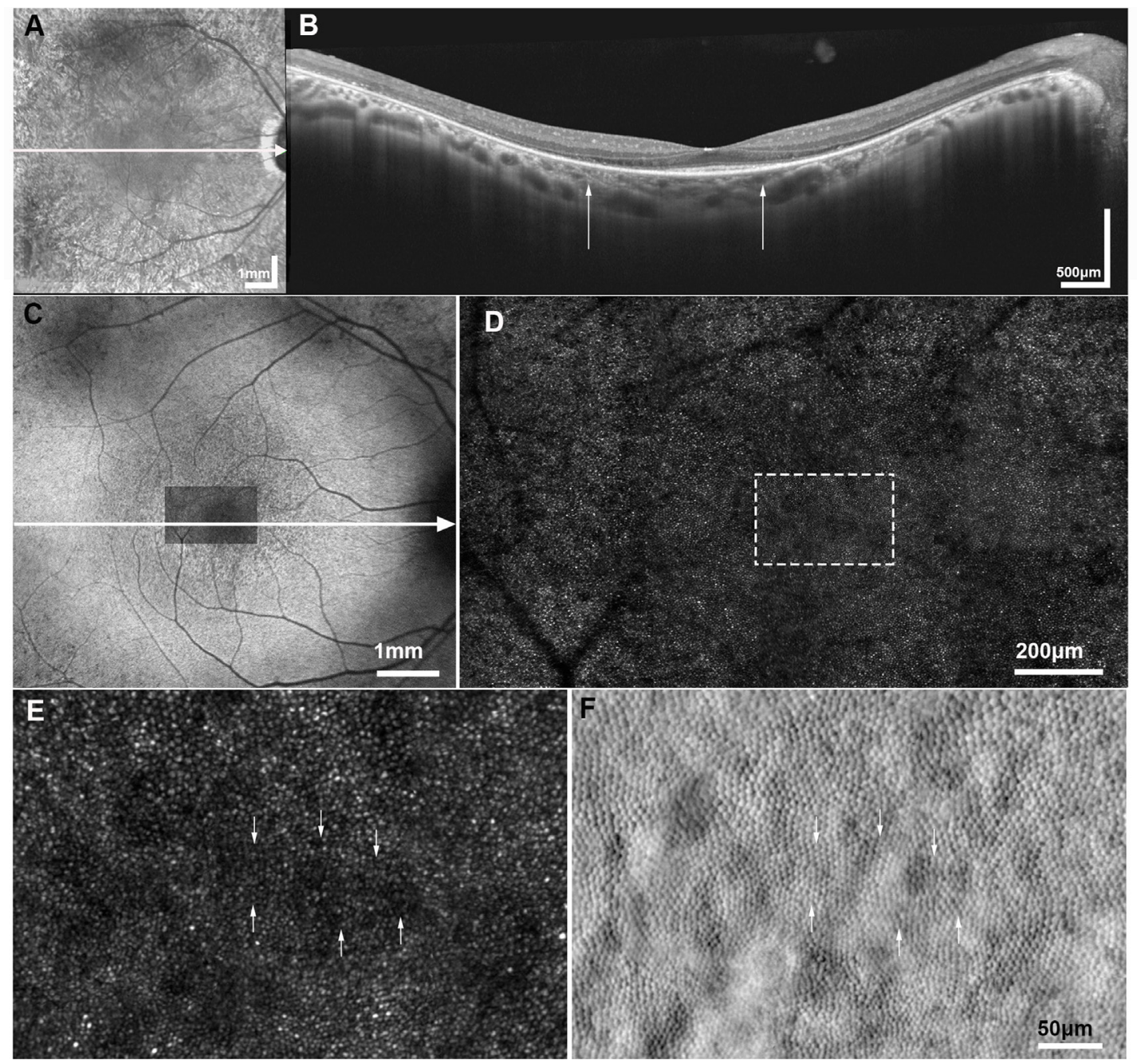

Figure 3 Multimodal imaging of retinitis pigmentosa. (A) Infrared reflectance (IR) fundus photograph of a subject (MM_0205) with X-linked retinitis pigmentosa associated with $R P G R$ gene. The white arrow represents the section of the optical coherence tomography (OCT) presented in (B). (B) Horizontal transfoveal OCT line scan, with the white arrows indicating the width of the corresponding AOSLO imaged area in (D). (C) Fundus autofluorescence imaging, with the confocal AOSLO (CAOSLO) imaged area (D) superimposed over the foveal avascular zone and the white arrow represents the section presented in the OCT scan (B). (D) CAOSLO imaging revealing a disrupted waveguiding mosaic, not as uniform in appearance as in a healthy subject (figure 1). (E) Magnification of CAOSLO over the estimated foveal centre (marked with a white dashed square in (D)) shows irregularly waveguiding cones, which appear dim (some are indicated with white arrows); and (F) the corresponding split detection AOSLO in exact spatial registration showing relatively healthy-appearing cone inner segments, the white arrows indicate the corresponding inner segments for the irregularly waveguiding cones identified with white arrows in (E). 
affected in each condition and the localisation of the affected protein either in the connecting cilium or outer segment. ${ }^{21}$

\section{Cone and cone-rod dystrophies \\ Cone-rod dystrophy}

Using an $\mathrm{AO}$ flood-illuminated ophthalmoscope ${ }^{76}$ one subject has been imaged showing a lack of waveguiding cones within clinically apparent atrophic regions and a contiguous cone mosaic elsewhere with enlarged cones and reduced peak cone densities. A correlation was observed between cone densities and multifocal ERG (mfERG) peak amplitudes. Similar observations, including structure-function correlation with mfERG, have been observed in three further studies of nine subjects in total. ${ }^{69} 7778$ AOSLO cone spacing measurements also correlated significantly with mfERG amplitude, retinal sensitivity and VA. ${ }^{6976-78}$

\section{Cone dystrophy with supernormal rod responses}

Vincent $e t$ al assessed macular morphology in cone dystrophy with supernormal rod responses with cAOSLO and reported decreased cone densities and a disrupted mosaic, with cones in groups, surrounded by patches of absent or non-waveguiding cones. ${ }^{79}$ It will be of interest to further investigate subjects with SD-AOSLO to probe whether the non-waveguiding cones are indeed absent or whether inner segments are present.

\section{Chorioretinal dystrophies \\ Choroideremia}

Patchy cone loss in symptomatic carriers and a normal photoreceptor mosaic in asymptomatic carriers have been observed. ${ }^{80}$ Disrupted parafoveal mosaics, with increased cone spacing, were seen in affected men, with more regular spacing near the borders of atrophy. ${ }^{80}$ In combination with OCT findings, likely simultaneous degeneration of the RPE and photoreceptors was suggested. The largest multimodal study to date including the use of CAOSLO ${ }^{81}$ describes a relatively intact central retina with a normal or reduced cone density at $0.5 \mathrm{~mm}$ eccentricity; and an abrupt loss of cones at the border of RPE atrophy, as well as hyper-reflective clumps of cones in younger patients $(<30$ years) and bubble-like lesions within the choroid; findings also identified by Sun et al. ${ }^{22}$ No RPE cells were visible in areas of cone loss, with IZ dropout preceding EZ disruption. ${ }^{81}$ Investigators thereby proposed that choroideremia (CHM) is primarily an RPE disorder followed by photoreceptor degeneration, with implications for intervention and the ongoing debate on cellular pathogenesis of CHM.

Only by using non-confocal SD-AOSLO ${ }^{22}$ has reliable visualisation of cones been possible in the bordering areas of atrophy, with abnormal and heterogeneous morphology, density and diameter. The cone mosaic terminates sharply before those areas, in direct contrast to previously reported RP transition zone imaging, which may relate to the likely primary RPE pathology in CHM. This study also concluded that RPE degeneration precedes photoreceptor loss.

These studies have resulted in AOSLO being incorporated in many ongoing CHM gene therapy trials and natural history studies. ${ }^{22} 8081$

\section{DISCUSSION AND FUTURE POSSIBILITIES}

IRDs are the most genetically and phenotypically heterogeneous conditions in medicine, with certain genotypes being extremely rare making it challenging to establish large informative cohorts, suggesting the need for multicentre studies. Many of the studies incorporated in our review highlight the need for longitudinal monitoring. Insights into disease progression are of great value including identification of the optimal therapeutic window and participant stratification.

AO ophthalmoscopy offers invaluable identification of structural detail on a cellular level, with several studies described herein exploring correlation between structure and function. Evolving AO-guided retinal sensitivity assessments ('nanoperimerty') will better allow correlation between cellular imaging and functional testing with exquisite retinotopic precision. ${ }^{82-84}$

One major common limitation is the challenge in imaging patients with nystagmus (eg, ACHM) and/or poor fixation (eg, STGD). Eye tracking systems incorporated into $\mathrm{AO}$ systems $\mathrm{s}^{85-87}$ can allow imaging of more subjects and improve data acquisition. Moreover, image processing and analysis are substantial bottlenecks that developments in, for example, machine learning, will hopefully solve in the near future, thereby allowing a broader application of $\mathrm{AO}$ technology.

While our review concentrates on IRDs, AO has been applied to many other conditions including albinism, ${ }^{14}$ age-related macular degeneration, ${ }^{88} 89$ diabetic retinopathy ${ }^{90}$ and autoimmune retinopathy, ${ }^{91}$ and also in basic and applied research, including facilitating insights in visual system neurophysiology. ${ }^{92}$

\section{CONCLUSIONS}

$\mathrm{AO}$ is a rapidly evolving field, which has a place in diagnosis, advice on prognosis, monitoring and management of IRDs. It can also probe underlying pathophysiology and facilitate improved understanding of cellular retinal anatomy and biology. We anticipate an increasing use of $\mathrm{AO}$ systems in the future due to the complementary information they provide compared with other imaging modalities and the ability to target functional measurements on individual cells, with particular application in longitudinal natural history studies and ongoing/planned interventional trials both for participant selection and monitoring treatment safety and efficacy.

\section{METHODS OF LITERATURE SEARCH}

PubMed was searched for articles related to AO and IRDs up to June 2017 with key words: Adaptive Optics, AO, AOSLO, Retinal Imaging individually and in combination with the conditions' name (eg, Achromatopsia) as well as their abbreviations (eg, ACHM).

Contributors MG drafted the manuscript and provided critical revision. MM conceived, supervised and revised the manuscript. AK, EJP, JC and AD provided critical revision of the manuscript.

Funding This work was supported by grants from the National Institute for Health Research Biomedical Research Centre at Moorfields Eye Hospital National Health Service Foundation Trust and UCL Institute of Ophthalmology, Fight for Sight (UK), The Macular Society (UK), Moorfields Eye Hospital Special Trustees, Moorfields Eye Charity, Retinitis Pigmentosa Fighting Blindness, The Wellcome Trust (099173/Z/12/Z) and the Foundation Fighting Blindness (USA). Research reported in this publication was supported in part by the National Eye Institute of the National Institutes of Health under award numbers U01 EY025477 and R01 EY017607. The content is solely the responsibility of the authors and does not necessarily represent the official views of the National Institutes of Health.

\section{Competing interests None declared.}

Provenance and peer review Not commissioned; externally peer reviewed.

Open access This is an open access article distributed in accordance with the terms of the Creative Commons Attribution (CC BY 4.0) license, which permits others to distribute, remix, adapt and build upon this work, for commercial use, provided the original work is properly cited. See: http://creativecommons.org/licenses/by/4.0/

(c) Article author(s) (or their employer(s) unless otherwise stated in the text of the article) 2018. All rights reserved. No commercial use is permitted unless otherwise expressly granted. 


\section{REFERENCES}

1 Liew G, Michaelides M, Bunce C. A comparison of the causes of blindness certifications in England and Wales in working age adults (16-64 years), 1999-2000 with 2009-2010. BMJ Open 2014;4:e004015.

2 Smith J, Ward D, Michaelides M, et al. New and emerging technologies for the treatment of inherited retinal diseases: a horizon scanning review. Eye 2015;29:1131-40.

3 Huang D, Swanson EA, Lin CP, et al. Optical coherence tomography. Science 1991:254:1178-81.

4 de Boer JF, Leitgeb R, Wojtkowski M. Twenty-five years of optical coherence tomography: the paradigm shift in sensitivity and speed provided by Fourier domain OCT [Invited]. Biomed Opt Express 2017;8:3248-80.

5 Walsh G, Charman WN, Howland HC. Objective technique for the determination of monochromatic aberrations of the human eye. Journal of the Optical Society of America. A, Optics and image science 1984;1:987-92.

6 Liang J, Williams DR, Miller DT. Supernormal vision and high-resolution retinal imaging through adaptive optics. Journal of the Optical Society of America. A, Optics, image science, and vision 1997:14:2884-92.

7 Roorda A, Williams DR. The arrangement of the three cone classes in the living human eye. Nature 1999:397:520-2.

8 Dubra A, Sulai Y, Norris JL, et al. Noninvasive imaging of the human rod photoreceptor mosaic using a confocal adaptive optics scanning ophthalmoscope. Biomed Opt Express 2011;2:1864-76

9 Rossi EA, Chung M, Dubra A, et al. Imaging retinal mosaics in the living eye. Eye 2011;25:301-8.

10 Merino D, Loza-Alvarez P. Adaptive optics scanning laser ophthalmoscope imaging: technology update. Clin Ophthalmol 2016;10:743-55.

11 Roorda A, Duncan JL. Adaptive optics ophthalmoscopy. Annu Rev Vis Sci 2015:1:19-50.

12 Roorda A, Williams DR. Optical fiber properties of individual human cones. J Vis 2002;2:4-12.

13 Zhang T, Godara P, Blanco ER, et al. Variability in human cone topography assessed by adaptive optics scanning laser ophthalmoscopy. Am J Ophthalmol 2015; 160:290-300.

14 Wilk MA, McAllister JT, Cooper RF, et al. Relationship between foveal cone specialization and pit morphology in albinism. Invest Ophthalmol Vis Sci 2014:55:4186-98.

15 Cooper RF, Dubis AM, Pavaskar A, et al. Spatial and temporal variation of rod photoreceptor reflectance in the human retina. Biomed Opt Express 2011;2:2577-89.

16 Merino D, Duncan JL, Tiruveedhula P, et al. Observation of cone and rod photoreceptors in normal subjects and patients using a new generation adaptive optics scanning laser ophthalmoscope. Biomed Opt Express 2011;2:2189-201.

17 Scoles D, Sulai YN, Langlo CS, et al. In vivo imaging of human cone photoreceptor inner segments. Invest Ophthalmol Vis Sci 2014;55:4244-51.

18 Dubis AM, Cooper RF, Aboshiha J, et al. Genotype-dependent variability in residual cone structure in achromatopsia: toward developing metrics for assessing cone health. Invest Ophthalmol Vis Sci 2014;55:7303-11.

19 Razeen MM, Cooper RF, Langlo CS, et al. Correlating Photoreceptor Mosaic Structure to Clinical Findings in Stargardt Disease. Trans/ Vis Sci Technol 2016;5:6.

20 Scoles D, Flatter JA, Cooper RF, et al. Assessing photoreceptor structure associated with ellipsoid zone disruptions visualized with optical coherence tomography. Retina 2016:36:91-103.

21 Sun LW, Johnson RD, Langlo CS, et al. Assessing Photoreceptor Structure in Retinitis Pigmentosa and Usher Syndrome. Invest Ophthalmol Vis Sci 2016;57:2428-42.

22 Sun LW, Johnson RD, Williams V, et al. Multimodal Imaging of Photoreceptor Structure in Choroideremia. PLoS One 2016;11:e0167526.

23 Scoles D, Sulai YN, Cooper RF, et al. Photoreceptor inner segment morphology in best vitelliform macular dystrophy. Retina 2017;37:741-8.

24 Langlo CS, Erker LR, Parker M, et al. Repeatability and longitudinal assessment of foveal cone structure in cngb3-associated achromatopsia. Retina 2017;37:1956-66.

25 Abozaid MA, Langlo CS, Dubis AM, et al. Reliability and repeatability of cone density measurements in patients with congenital achromatopsia. Adv Exp Med Biol 2016:854:277-83

26 Cooper RF, Sulai YN, Dubis AM, et al. Effects of intraframe distortion on measures of cone mosaic geometry from adaptive optics scanning light ophthalmoscopy. Trans/ Vis Sci Technol 2016;5:10.

27 Curcio CA, Sloan KR, Kalina RE, et al. Human photoreceptor topography. J Comp Neurol 1990;292:497-523.

28 Cooper RF, Wilk MA, Tarima S, et al. Evaluating Descriptive Metrics of the Human Cone Mosaic. Invest Ophthalmo/ Vis Sci 2016;57:2992-3001.

29 Garrioch R, Langlo C, Dubis AM, et al. Repeatability of in vivo parafoveal cone density and spacing measurements. Optom Vis Sci 2012;89:632-43.

30 Cooper RF, Lombardo M, Carroll J, et al. Methods for investigating the local spatial anisotropy and the preferred orientation of cones in adaptive optics retinal images. Vis Neurosci 2016:33:E005.

31 Zhang Y, Rha J, Jonnal R, et al. Adaptive optics parallel spectral domain optical coherence tomography for imaging the living retina. Opt Express 2005;13:4792-811.
32 Tanna P, Strauss RW, Fujinami K, et al. Stargardt disease: clinical features, molecular genetics, animal models and therapeutic options. Br J Ophthalmol 2017;101:25-30.

33 Chen Y, Ratnam K, Sundquist SM, et al. Cone photoreceptor abnormalities correlate with vision loss in patients with Stargardt disease. Invest Ophthalmol Vis Sci 2011:52:3281-92.

34 Song H, Rossi EA, Latchney L, et al. Cone and rod loss in Stargardt disease revealed by adaptive optics scanning light ophthalmoscopy. JAMA Ophthalmol 2015;133:1198-203.

35 Tanna P, Kasilian M, Strauss R, et al. Reliability and repeatability of cone density measurements in patients with stargardt disease and RPGR-associated retinopathy. Invest Ophthalmol Vis Sci 2017:58:3608-15.

36 Scholl HP, Strauss RW, Singh MS, et al. Emerging therapies for inherited retinal degeneration. Sci Trans/ Med 2016;8:368rv6.

37 Kong X, Strauss RW, Cideciyan AV, et al. Visual acuity change over 12 months in the prospective progression of atrophy secondary to stargardt disease (progstar) study: progstar report number 6. Ophthalmology 2017;124:1640-51.

38 Kay DB, Land ME, Cooper RF, et al. Outer retinal structure in best vitelliform macular dystrophy. JAMA Ophthalmol 2013;131:1207-15.

39 Duncan JL, Ratnam K, Birch DG, et al. Abnormal cone structure in foveal schisis cavities in X-linked retinoschisis from mutations in exon 6 of the RS1 gene. Invest Ophthalmol Vis Sci 2011;52:9614-23.

40 Aboshiha J, Dubis AM, Carroll J, et al. The cone dysfunction syndromes. Br J Ophthalmol 2016;100:115-21.

41 Carroll J, Choi SS, Williams DR. In vivo imaging of the photoreceptor mosaic of a rod monochromat. Vision Res 2008:48:2564-8.

42 Genead MA, Fishman GA, Rha J, et al. Photoreceptor structure and function in patients with congenital achromatopsia. Invest Ophthalmol Vis Sci 2011;52:7298-308.

43 Sundaram V, Wilde C, Aboshiha J, et al. Retinal structure and function in achromatopsia: implications for gene therapy. Ophthalmology 2014;121:234-45.

44 Ueno S, Nakanishi A, Kominami T, et al. In vivo imaging of a cone mosaic in a patient with achromatopsia associated with a GNAT2 variant. Jpn J Ophthalmol 2017;61:92-8

45 Langlo CS, Patterson EJ, Higgins BP, et al. Residual Foveal Cone Structure in CNGB3Associated Achromatopsia. Invest Ophthalmol Vis Sci 2016;57:3984-95.

46 Carroll J, Rossi EA, Porter J, et al. Deletion of the X-linked opsin gene array locus control region (LCR) results in disruption of the cone mosaic. Vision Res 2010:50:1989-99.

47 Carroll J, Dubra A, Gardner JC, et al. The effect of cone opsin mutations on retinal structure and the integrity of the photoreceptor mosaic. Invest Ophthalmol Vis Sci 2012;53:8006-15

48 Patterson EJ, Kasilian M, Kalitzeos A, et al. "Assessing cone photoreceptor structure in patients with mutations in the OPN1LW/OPN1MW gene array". Invest. Ophthalmol. Vis. Sci 2017:E-Abstract 1257.

49 Cideciyan AV, Hufnagel RB, Carroll J, et al. Human cone visual pigment deletions spare sufficient photoreceptors to warrant gene therapy. Hum Gene The 2013:24:993-1006.

50 Curcio CA, Allen KA, Sloan KR, et al. Distribution and morphology of human cone photoreceptors stained with anti-blue opsin. J Comp Neurol 1991:312:610-24.

51 Michaelides M, Rha J, Dees EW, et al. Integrity of the cone photoreceptor mosaic in oligocone trichromacy. Invest Ophthalmol Vis Sci 2011;52:4757-64.

52 Strauss RW, Dubis AM, Cooper RF, et al. Retinal architecture in RGS9- and R9APassociated retinal dysfunction (bradyopsia). Am J Ophthalmol 2015:160:1269-75.

53 Michaelides M, Li Z, Rana NA, et al. Novel mutations and electrophysiologic findings in RGS9- and R9AP-associated retinal dysfunction (Bradyopsia). Ophthalmology 2010:117:120-7.

54 Schwartz M, Haim M, Skarsholm D. X-linked myopia: bornholm eye disease. linkage to DNA markers on the distal part of Xq. Clin Genet 1990;38:281-6.

55 Young TL, Deeb SS, Ronan SM, et al. X-linked high myopia associated with cone dysfunction. Arch Ophthalmol 2004;122:897-908.

56 Michaelides M, Johnson S, Bradshaw K, et al. X-linked cone dysfunction syndrome with myopia and protanopia. Ophthalmology 2005;112:1448-54.

57 Guo X, Xiao X, Li S, et al. Nonsyndromic high myopia in a Chinese family mapped to MYP1: linkage confirmation and phenotypic characterization. Arch ophthalmol 2010;128:1473-9.

58 Li J, Gao B, Guan L, et al. Unique variants in OPN1LW cause both syndromic and nonsyndromic $x$-linked high myopia mapped to MYP1. Invest Ophthalmol Vis Sci 2015;56:4150-5.

59 Neitz J, Wagner-Schuman M, Dubra A, et al Cone mosaic disruption caused by L/M opsin mutations in bornholm eye disease. Invest Ophthalmol Vis Sci 2011:52:4896-96

60 Patterson EJ, Wilk M, Langlo CS, et al. Cone photoreceptor structure in patients with $x$-linked cone dysfunction and red-green color vision deficiency. Invest Ophthalmol Vis Sci 2016:57:3853-63.

61 Godara P, Cooper RF, Sergouniotis PI, et al. Assessing retinal structure in complete congenital stationary night blindness and Oguchi disease. Am J Ophthalmol 2012;154:987-1001 
62 Dryja TP. Molecular genetics of Oguchi disease, fundus albipunctatus, and other forms of stationary night blindness: LVII Edward Jackson Memorial Lecture. Am J Ophthalmol 2000;130:547-63.

63 Weiss ER, Ducceschi MH, Horner TJ, et al. Species-specific differences in expression of G-protein-coupled receptor kinase (GRK) 7 and GRK1 in mammalian cone photoreceptor cells: implications for cone cell phototransduction. J Neurosci 2001;21:9175-84.

64 Song $H$, Latchney L, Williams D, et al. Fluorescence adaptive optics scanning laser ophthalmoscope for detection of reduced cones and hypoautofluorescent spots in fundus albipunctatus. JAMA Ophthalmo/ 2014;132:1099-104.

65 Makiyama Y, Ooto S, Hangai M, et al. Cone abnormalities in fundus albipunctatus associated with RDH5 mutations assessed using adaptive optics scanning laser ophthalmoscopy. Am J Ophthalmol 2014;157:e1-4:558-70.

66 Tee JJ, Smith AJ, Hardcastle AJ, et al. RPGR-associated retinopathy: clinical features, molecular genetics, animal models and therapeutic options. Br J Ophthalmol 2016;100:1022-7.

67 Fishman GA, Weinberg AB, McMahon TT. X-linked recessive retinitis pigmentosa. clinical characteristics of carriers. Arch Ophthalmol 1986;104:1329-35.

68 Makiyama Y, Ooto S, Hangai M, et al. Macular cone abnormalities in retinitis pigmentosa with preserved central vision using adaptive optics scanning laser ophthalmoscopy. PLoS One 2013;8:e79447.

69 Duncan JL, Zhang Y, Gandhi J, et al. High-resolution imaging with adaptive optics in patients with inherited retinal degeneration. Invest Ophthalmol Vis Sci 2007:48:3283-91.

70 Park SP, Lee W, Bae EJ, et al. Early structural anomalies observed by high-resolution imaging in two related cases of autosomal-dominant retinitis pigmentosa. Ophthalmic Surg Lasers Imaging Retina 2014;45:469-73.

71 Talcott KE, Ratnam K, Sundquist SM, et al. Longitudinal study of cone photoreceptors during retinal degeneration and in response to ciliary neurotrophic factor treatment. Invest Ophthalmol Vis Sci 2011;52:2219-26.

72 Zayit-Soudry S, Sippl-Swezey N, Porco TC, et al. Repeatability of cone spacing measures in eyes with inherited retinal degenerations. Invest Ophthalmol Vis Sci 2015;56:6179-89.

73 Ratnam K, Carroll J, Porco TC, et al. Relationship between foveal cone structure and clinical measures of visual function in patients with inherited retinal degenerations. Invest Ophthalmol Vis Sci 2013;54:5836-47.

74 Birch DG, Weleber RG, Duncan JL, et al. Randomized trial of ciliary neurotrophic factor delivered by encapsulated cell intraocular implants for retinitis pigmentosa. Am J Ophthalmol 2013;156:283-92.

75 Ratnam K, Västinsalo H, Roorda A, et al. Cone structure in patients with usher syndrome type III and mutations in the Clarin 1 gene. JAMA Ophthalmol 2013:131:67-74.
76 Wolfing Jl, Chung M, Carroll J, et al. High-resolution retinal imaging of cone-rod dystrophy. Ophthalmology 2006;113:e1:1014-9.

77 Choi SS, Doble N, Hardy JL, et al. In vivo imaging of the photoreceptor mosaic in retinal dystrophies and correlations with visual function. Invest Ophthalmo/ Vis SC 2006;47:2080-92.

78 Roorda A, Zhang Y, Duncan JL. High-resolution in vivo imaging of the RPE mosaic in eyes with retinal disease. Invest Ophthalmol Vis Sci 2007;48:2297-303.

79 Vincent A, Wright T, Garcia-Sanchez Y, et al. Phenotypic characteristics including in vivo cone photoreceptor mosaic in KCNV2-related "cone dystrophy with supernormal rod electroretinogram". Invest Ophthalmol Vis Sci 2013;54:898-908.

80 Syed R, Sundquist SM, Ratnam K, et al. High-resolution images of retinal structure in patients with choroideremia. Invest Ophthalmol Vis Sci 2013;54:950-61.

81 Morgan Jl, Han G, Klinman E, et al. High-resolution adaptive optics retinal imaging of cellular structure in choroideremia. Invest Ophthalmol Vis Sci 2014;55:6381-97.

82 Tuten WS, Tiruveedhula P, Roorda A. Adaptive optics scanning laser ophthalmoscopebased microperimetry. Optom Vis Sci 2012;89:563-74.

83 Bruce KS, Harmening WM, Langston BR, et al. Normal perceptual sensitivity arising from weakly reflective cone photoreceptors. Invest Ophthalmol Vis SCi 2015;56:4431-8

84 Wang Q, Tuten WS, Lujan BJ, et al. Adaptive optics microperimetry and OCT images show preserved function and recovery of cone visibility in macular telangiectasia type 2 retinal lesions. Invest Ophthalmol Vis Sci 2015;56:778-86.

85 Sheehy CK, Tiruveedhula P, Sabesan R, et al. Active eye-tracking for an adaptive optics scanning laser ophthalmoscope. Biomed Opt Express 2015;6:2412-23.

86 Privitera CM, Sabesan R, Winter S, et al. Eye-tracking technology for real-time monitoring of transverse chromatic aberration. Opt Lett 2016;41:1728-31.

87 Tam J, Roorda A. Speed quantification and tracking of moving objects in adaptive optics scanning laser ophthalmoscopy. J Biomed Opt 2011;16:036002.

88 Gocho K, Sarda V, Falah S, et al. Adaptive optics imaging of geographic atrophy. Invest Ophthalmol Vis Sci 2013;54:3673-80.

89 Querques G, Kamami-Levy C, Georges A, et al. Adaptive optics imaging of foveal sparing in geographic atrophy secondary to age-related macular degeneration. Retina 2016;36:247-54

90 Burns SA, Elsner AE, Chui TY, et al. In vivo adaptive optics microvascular imaging in diabetic patients without clinically severe diabetic retinopathy. Biomed Opt Express 2014;5:961-74

91 Davoudi S, Ebrahimiadib N, Yasa C, et al. Outcomes in autoimmune retinopathy patients treated with rituximab. Am J Ophthalmo/ 2017;180:124-32.

92 Marcos S, Werner JS, Burns SA, et al. Vision science and adaptive optics, the state of the field. Vision Res 2017:132:3-33. 